\title{
PROPERTIES OF AN UNUSUAL GENETIC ELEMENT IN STAPHYLOCOCCUS AUREUS
}

\author{
R. W. LACEY \\ Department of Bacteriology, King's Lynn General Hospital, London Road, King's Lynn, \\ Norfolk PE30 5QD
}

DURING a study of recently isolated methicillin-resistant strains of Staphylococcus aureus we detected the presence in one isolate (strain M7) of linked genes determining production of penicillinase and resistance to neomycin, streptomycin and cadmium ions. Study of this element has revealed some unusual findings, including its transfer between certain cultures at frequencies as high as $10^{-3}$, and its transfer by transduction from lysates at frequencies of up to $10^{-2}$. This paper describes observations that may be relevant to the evolution of $S$. aureus, including factors affecting transfer of antibiotic resistance between strains.

\section{MATERIALS AND METHODS}

Experimental procedures. Details of the experimental procedures used in this study including media, determination of antibiotic sensitivity, UV induction, transduction, transfer of resistance in mixed cultures, replica plating and bacteriophage typing are described elsewhere (Lacey, 1971 a) but the design of experiments is summarised at appropriate points in the Results section.

Organisms and resistance transfer. Strain 609 tet-r contains a stable plasmid specifying tetracycline resistance (Lacey, 1971a), and has lost its original neomycin-resistance determinant. The derivatives of strain 6936 used were 6936 nov-r, 6936 nov-r rif-r, and 6936 nov-r rif-r fus-r (nov-r denotes chromosomal resistance to novobiocin, rif-r, resistance to rifampicin, and fus-r resistance to fusidic acid). Strain M7-wild is a multi-resistant culture isolated in King's Lynn during 1978 and 1030 rif- $r$ is a non-lysogenic strain (Lacey, 1971a), containing a chromosomal mutation to rifampicin resistance. It was necessary to use a variety of recipients for detection of transfer through successive clones of a strain. The $\operatorname{Rec}^{-}$strain RN 981 was supplied by Dr R.P. Novick of New York.

Resistance transfer was detected by incorporation of two of the following agents into nutrient agar: cadmium acetate $10^{-4} \mathrm{M}$, neomycin $2.5 \mu \mathrm{g} / \mathrm{ml}$, streptomycin $10 \mu \mathrm{g} / \mathrm{ml}$, tetracycline 2.5 $\mu \mathrm{g} / \mathrm{ml}$, novobiocin $2.5 \mu \mathrm{g} / \mathrm{ml}$, rifampicin $10 \mu \mathrm{g} / \mathrm{ml}$, fusidic acid $5 \mu \mathrm{g} / \mathrm{ml}$. The proportion of recipient and donor cells in a culture was determined by spreading dilutions of the culture on milk agar that sometimes contained a selective antibiotic. Transfer frequencies were calculated from parallel experiments performed in triplicate; maximum variability between these was fourfold.

Definitions. The genetic element from strain $\mathrm{M} 7$ determining production of penicillinase (P) resistance to cadmium ions $(\mathrm{C})$, neomycin $(\mathrm{N})$ kanamaycin and streptomycin $(\mathrm{S})$ is referred to as plasmid (CPNS) and its fragments as (CP) and (NS).

\section{RESULTS}

Phenotypic properties determined by plasmid (CPNS)

The properties coded by plasmid (CPNS) were determined after its transfer

Received 17 Oct. 1978; accepted 10 Jan. 1979. 
to strain 6936. The resistance of 20 derivatives that possessed the full resistance traits are shown in table $I$. This plasmid determines resistance to cadmium ions but not to arsenate or mercury. Five colonies of strain 6936 and of strain 609, each harbouring the plasmid (CPNS) showed phage-typing patterns identical to that of the corresponding wild strains.

TABLE I

Phenotypic properties determined by plasmid (CPNS)

\begin{tabular}{l|cc}
\hline Antibacterial agent & Sensitivity/resistance* & $\begin{array}{c}\text { Minimum } \\
\text { inhibitory } \\
\text { concentration }\end{array}$ \\
\hline Cadmium acetate & $\mathbf{R}$ & $>10^{-4} \mathrm{M}$ \\
Penicillin & $\mathbf{R} \dagger$ & $\ldots$ \\
Sodium arsenate & $\mathbf{S}$ & $250 \mu \mathrm{g} / \mathrm{ml}$ \\
Mercurous chloride & $\mathbf{S}$ & $25 \mu \mathrm{g} / \mathrm{ml}$ \\
Neomycin & $\mathbf{R}$ & $50 \mu \mathrm{g} / \mathrm{ml}$ \\
Kanamycin & $\mathbf{R}$ & $400 \mu \mathrm{g} / \mathrm{ml}$ \\
Streptomycin & $\mathbf{R}$ & $100 \mu \mathrm{g} / \mathrm{ml}$ \\
\hline
\end{tabular}

* $\mathrm{S}=$ sensitive, $\mathrm{R}=$ resistant.

$\dagger$ Resistance due to production of penicillinase.

$\ldots=$ not tested.

\section{Loss of plasmid (CPNS) from strain M7}

Evidence for loss of plasmid (CPNS) from thrice-purified cultures of strain M7 was obtained after growth at $37^{\circ} \mathrm{C}$ for 7 days, or after storage for 3 months at room temperature. These cultures were then inoculated into nutrient broth and incubated overnight. Loss of properties determined by (CPNS) was detected by replica plating on medium containing cadmium acetate $10^{-4} \mathrm{M}$ or neomycin $2.5 \mu \mathrm{g} / \mathrm{ml}$. When loss of resistance occurred, there was always coincidental loss of penicillinase production, and resistance to cadmium ions and neomycin. After storage at room temperature, 1240 colonies were examined and 72 were found to have lost these properties; after incubation at $37^{\circ} \mathrm{C}$, of 378 colonies examined 21 had lost these traits. Ten colonies of each of strain $\mathrm{M} 7\left(\mathrm{CPNS}^{-}\right)$obtained under each condition was examined further (table II). Thus, phenotypic properties associated with gain of plasmid (CPNS)

TABLE II

Phenotypic properties shown by strain M7 after loss of plasmid (CPNS)

\begin{tabular}{|c|c|c|c|c|c|}
\hline \multirow{2}{*}{ Strain } & \multirow{2}{*}{$\begin{array}{l}\text { Penicillinase } \\
\text { production }\end{array}$} & \multicolumn{4}{|c|}{ Minimum inhibitory concentration of } \\
\hline & & $\begin{array}{l}\text { streptomycin } \\
(\mathrm{mg} / \mathrm{ml})\end{array}$ & $\begin{array}{l}\text { neomycin } \\
(\mu \mathrm{g} / \mathrm{ml})\end{array}$ & $\underset{(\mu \mathrm{g} / \mathrm{ml})}{\text { kanamycin }}$ & cadmium ions \\
\hline M7-wild & + & 100 & 50 & 1600 & $>10^{-4} M$ \\
\hline $\begin{array}{l}\left.\text { M7(CPNS }{ }^{-}\right) \text {, obtained on storage } \\
\text { M7(CPNS }^{-} \text {), after incubation at } 37^{\circ} \mathrm{C}^{*}\end{array}$ & - & $\begin{array}{l}100 \\
100\end{array}$ & $\begin{array}{l}0 \cdot 5 \\
0 \cdot 5\end{array}$ & $\begin{array}{l}100 \\
100\end{array}$ & $\begin{array}{l}1.5 \times 10^{-6} \mathrm{M} \\
1.5 \times 10^{-6} \mathrm{M}\end{array}$ \\
\hline
\end{tabular}

* 10 colonies of each examined. 
corresponded to those associated with the loss of this putative plasmid, except for high-level streptomycin resistance that is determined by strain M7-wild and M7 $\left(\mathrm{CPNS}^{-}\right)$, and differences in the level of kanamycin resistance. These findings support the conclusion that strain M7 contain two sets of genes determining resistance to streptomycin and kanamycin; one set is carried by the (CPNS) plasmid determining an MIC of $400 \mu \mathrm{g} / \mathrm{ml}$ for kanamycin, and the other is at an uncertain locus determining an MIC of $100 \mu \mathrm{g} / \mathrm{ml}$ for kanamycin. The combined phenotypic effect is to produce an MIC for kanamycin of 1600 $\mu \mathrm{g} / \mathrm{ml}$. Of strain $\mathrm{M}^{7}\left(\mathrm{CPNS}^{-}\right) 10$ cultures had a phage-typing pattern identical to that of strain $\mathrm{M} 7$-wild, i.e., phage type 77 .

\section{Transfer of plasmid (CPNS) through several strains of S. aureus}

Incubation of strain M7 with either strain 609 or 6936 in mixed culture with a selection for transfer of resistance to cadmium ions, neomycin or streptomycin, revealed that transfer occurred at low frequency $\left(c .10^{-8}\right)$. Replica plating of 120 resistant colonies demonstrated that the entire range of phenotypic properties attributable to plasmid (CPNS) had been acquired en bloc in 108 colonies. However, in the remaining 12 colonies, two other patterns of transfer were seen-either resistance to neomycin, streptomycin and kanamycin (four isolates), or resistance to cadmium ions accompanied by penicillinase production (eight isolates). These traits were present in isolates from both recipient strains, 609 and 6936. Two of these isolates were examined in detail, 609 (CP) which showed resistance to cadmium ions and production of penicillinase, and 609 (NS) which showed resistance to neomycin, streptomycin and kanamycin. Cultures 609 (CPNS), 609 (CP), and 609 (NS) were then investigated for the ability of their newly acquired resistance determinants to transfer to other cultures (table III). In general, the element (CPNS) was transferred at a higher frequency than $(\mathrm{CP})$, and in turn $(\mathrm{CP})$ was transferred at a higher frequency than (NS). The transfer of (CPNS) occurred at very high frequencies between strains 609 and 6936, and between different derivatives of strain 6936 $\left(1.3 \times 10^{-4}\right.$ and $1.5 \times 10^{-3}$ respectively). Transfer of (CPNS) and (CP) occurred at low frequency $\left(10^{-9}\right)$ from strain $\mathrm{M} 7$ wild. This low frequency of transfer was maintained when each of these elements was returned to a derivative of strain M7 that had lost the (CPNS) plasmid, designated M7 (CPNS ${ }^{-}$). The high frequencies obtained were therefore probably not due to host modification of the plasmid within strain 6936 or 609 . No transfer of the element (NS) to M7 (CPNS ${ }^{-}$) was detected by selection with neomycin $10 \mu \mathrm{g} / \mathrm{ml}$ and streptomycin $10 \mathrm{mg} / \mathrm{ml}$. These concentrations of antibiotic were essential for selecting for the acquisition of (CPNS) by M7 (CPNS ${ }^{-}$).

After the transfer of (CPNS) from donors other than strain M7, a total of 2100 colonies of strains 609 (CPNS), 6936 (CPNS) and 1030 (CPNS) were examined by replica plating for the possession of a full complement of phenotypic characters. Every colony examined possessed the complete set of characacters. Thus the postulated fragmentation of (CPNS) into (NS) and (CP) was probably a property of the donor, i.e., strain $\mathrm{M} 7$, rather than the recipient. 
TABLE III

Transfer of determinants (CPNS), (CP), and (NS) between strains in mixed culture, and by comventional transduction.

\begin{tabular}{|c|c|c|c|c|}
\hline Determinant & $\begin{array}{l}\text { Donor } \\
\text { strain }\end{array}$ & $\begin{array}{l}\text { Recipient } \\
\text { strain }\end{array}$ & $\begin{array}{l}\text { Frequency of transfer in } \\
\text { mixed culture* }\end{array}$ & $\begin{array}{l}\text { Frequency of transfer from } \\
\text { cell-free lysate } \dagger\end{array}$ \\
\hline \multirow[t]{2}{*}{$\begin{array}{l}\text { CPNS } \\
\text { CPNS } \\
\text { CPNS } \\
\text { CPNS } \\
\text { CPNS } \\
\text { CPNS } \\
\text { CPNS }\end{array}$} & \multirow{2}{*}{$\begin{array}{l}\text { M7 } \\
\text { M7 } \\
609 \\
6936 \\
6936 \\
6936 \\
\left.\text { M7(CPNS }^{-}{ }^{-} \text {(CPNS }^{+}\right)^{8} \\
\text { (CPNS }^{2}\end{array}$} & $\begin{array}{l}609 \\
6936 \\
6936 \\
6936 \\
1030 \\
\mathrm{M}^{\prime}\left(\mathrm{CPNS}^{-}\right)\end{array}$ & $\begin{array}{l}3.0 \times 10^{-9} \\
7.0 \times 10^{-9} \\
1.3 \times 10^{-4} \\
1.5 \times 10^{-3} \\
2.0 \times 10^{-8} \\
9.0 \times 10^{-6}\end{array}$ & $\begin{array}{c}\cdots \\
\cdots \\
8.0 \times 10^{-4} \\
3.4 \times 10^{-2} \\
7.9 \times 10^{-6} \\
6.0 \times 10^{-6}\end{array}$ \\
\hline & & 6936 & $3.6 \times 10^{-9}$ & $\ldots$ \\
\hline $\begin{array}{l}\text { CP } \\
C P \\
C P \\
C P \\
C P \\
C P \\
C P\end{array}$ & $\begin{array}{l}\text { M7 } \\
\text { M7 } \\
609 \\
6936 \\
6936 \\
6936 \\
M^{2}\left(\text { CPNS }^{-}\right)\end{array}$ & $\begin{array}{l}609 \\
6936 \\
6936 \\
6936 \\
1030 \\
\text { M7CPNS }^{-} \text {) }\end{array}$ & $\begin{array}{c}1.0 \times 10^{-9} \\
1.5 \times 10^{-9} \\
4.9 \times 10^{-7} \\
7.1 \times 10^{-7} \\
0 \ddagger \\
6.2 \times 10^{-7}\end{array}$ & $\begin{array}{c}\cdots \\
5.0 \times 10^{-5} \\
4.5 \times 10^{-5} \\
1.0 \times 10^{-7} \\
2.0 \times 10^{-6}\end{array}$ \\
\hline $\begin{array}{l}\text { NS } \\
\text { NS } \\
\text { NS } \\
\text { NS } \\
\text { NS } \\
\text { NS }\end{array}$ & $\begin{array}{l}\quad\left(\mathrm{CP}^{+}\right) \| \\
\mathrm{M} 7 \\
\mathrm{M} 7 \\
609 \\
6936 \\
6936 \\
6936\end{array}$ & $\begin{array}{r}6936 \\
609 \\
6936 \\
6936 \\
6936 \\
1030 \\
M^{7}\left(\mathrm{CPNS}^{-}\right)\end{array}$ & $\begin{array}{c}5.0 \times 10^{-8} \\
3.0 \times 10^{-9} \\
2.0 \times 10^{-9} \\
0 \\
0 \\
0 \\
0\end{array}$ & $\begin{array}{c}\cdots \\
\ldots \\
2.2 \times 10^{-7} \\
5.8 \times 10^{-7} \\
1.0 \times 10^{-8} \\
\ldots\end{array}$ \\
\hline
\end{tabular}

- Number of resistant recipients/total recipients after 24-h incubation (mean of 3 experiments). + Number of transductants/plaque-forming units of phage (unirradiated).

$\ddagger 0=<10^{-9}$

$\S \mathrm{M}^{7}$ (CPNS $\left.^{-}\right)\left(\mathrm{CPNS}^{+}\right.$) represents a strain of $\mathrm{M} 7$ that had first lost the (CPNS) plasmid and then reacquired it.

\| $\mathrm{M}^{7}\left(\mathrm{CPNS}^{-}\right)\left(\mathrm{CP}^{+}\right)$represents a strain of $\mathrm{M} 7$ that had first lost the (CPNS) plasmid and then acquired (CP).

$\ldots=$ Not tested.

Effect of the presence of fragment $(C P)$ and $(N S)$ in a recipient on its acquisition of (CPNS) in mixed culture

Strain 6936 (CPNS) was incubated in mixed culture with recipient strains 609, 609(CP), 609(NS), 6936, 6936(CP), 6936(NS). The frequency of transfer of (CPNS) was unaffected by the presence of (NS), and the two putative strains 609(NS) (CPNS) and 6936 (NS) (CPNS) could transfer subsequently the two elements seperately. However transfer of (CPNS) to either 609(CP) or 6936(CP) occurred at a much reduced frequency - a reduction to $0.1 \%$ - compared with that to cultures 609 and 6936. Attempts to transfer only the (CP) determinant from the cultures that were putatively 609(CP) (CPNS) and 6936(CP) (CPNS) failed. Presumably either only $\left(\mathrm{CP}^{-}\right)$recipients had acquired the incoming plasmid (CPNS), or the latter had "displace" (CP). Three colonies that possessed the phenotype 6936(NS) were obtained after incubation of strain 6936(CPNS) with strain 6936(CP). The element (NS) had presumably arisen in these clones by recombination. The (NS) determinant was considered to be chromosomal, giving features identical to that of 6936(NS) that had been obtained after incubation of M7-wild with 6936 (see below). 
Thus the possession of the fragment (CP) gives the cell immunity to incoming (CPNS) but (NS) does not.

Location of genes (CPNS), (CP) and (NS) in strain 6936

The location of each of these genes was identified by conventional genetic techniques-stability of the phenotypic traits, effect of ultraviolet irradiation of the lysate on the frequency of transduction and attempted transduction to a $\mathrm{Rec}^{-}$organism. Both (CPNS) and (CP) were unstable in strain 6936. Of 1179 colonies of 6936 (CPNS) tested after three culture cycles at $37^{\circ} \mathrm{C}$ in nutrient broth, 38 had lost all the phenotypic traits associated with the presence of (CPNS). Of 965 colonies of 6936(CP) so examined, five had lost the relevant

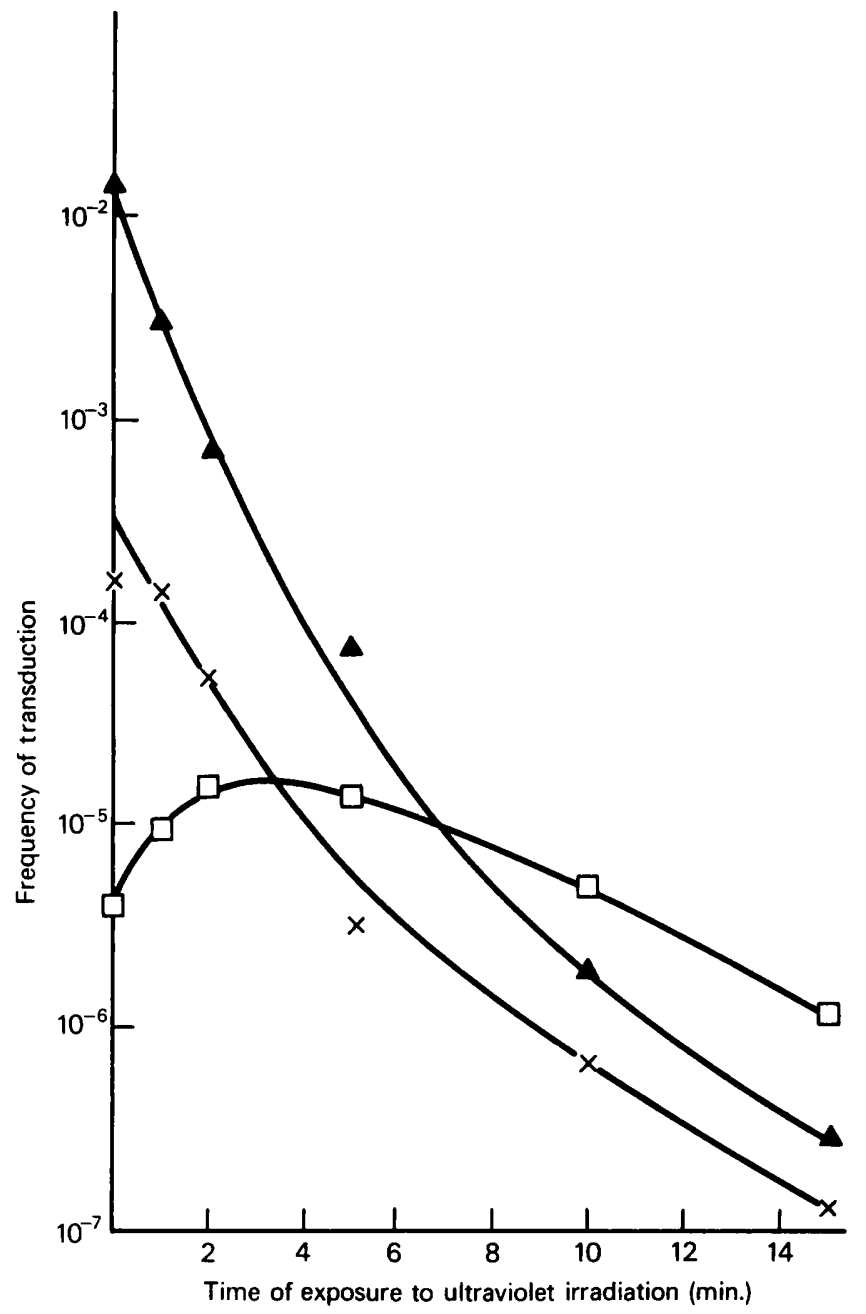

FIGURE. Effect of ultraviolet irradiation of lysates on the frequency of transfer of (CPNS), (CP) and (NS) by

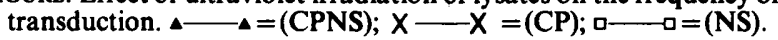


traits. In contrast, 2900 colonies of (CPNS)(NS) similarly tested, were all still resistant to neomycin and streptomycin.

The effect of ultraviolet irradiation on the transduction frequency of the elements from strains 6936(CPNS) and 6936(CP) revealed kinetics typical of plasmid inheritance. The frequencies for the elements (CPNS) and (CP) in unirradiated organisms were high (for (CPNS) as high as $10^{-2}$ ), with an exponential decay in the frequency after increasing exposure to ultraviolet light (figure). For transduction of the element (NS) from strain 6936 (NS) the transduction frequency was initially lower than that of the other elements, with a fivefold stimulation in the frequency with low doses of irradiation; these are characteristics of a chromosomal location for the genes (figure). Results of transduction to a $\mathrm{Rec}^{-}$strain were consistent with these conclusions about the gene; both the elements (CPNS) and (CP) could be transduced to a $\mathrm{Rec}^{-}$strain at frequencies of $9.4 \times 10^{-6}$ and $2.8 \times 10^{-7}$ respectively but (NS) could not (frequency $<10^{-9}$ ). It is concluded that (CPNS) and (CP) are plasmid determinants and that (NS) is chromosomal. A lysate of strain 6936(NS)(CP) containing the two fragments from the element (CPNS) was also used as a source of transducing particles. From this lysate the kinetics of transduction of (CP) was similar to that from $6936(\mathrm{CP})$, and that of (NS) was similar to that from $6936(\mathrm{SN})$.

\section{Correlation between transfer frequencies in mixed culture and transfer from cell-free lysates}

Each of the prospective donor cultures that contained the elements (CPNS), (CP) or (NS) (table III) was investigated for the ability of these genes to be transduced to recipients after ultraviolet induction. Strain M7 is noninducible and transduction frequencies cannot be calculated because no phage plaques can be detected. Lysates from almost all remaining cultures yielded transductants, although the frequencies were extremely variable, ranging from c. $10^{-2}$ to $10^{-8}$ (table III). As in mixed-culture incubation, the highest frequencies were obtained for (CPNS) from strain 6936 and the lowest frequencies were obtained for (NS) from several donors. In general, the correlation between the frequency of transfer in mixed culture and by conventional transduction was good, although the frequencies in mixed culture were somewhat lower than those from the lysates.

\section{Effect of lysogenisation of the recipient on frequency of transfer}

When a derivative of either strain M7 or strain 1030, neither of which contained a prophage capable of plaque formation, was used as recipient in mixed-culture experiments, the transfer frequency of the element (CPNS) was low regardless of the donor, whilst the frequency of transfer of (CPNS) to the lysogenic recipients 609 and 6936 was high (table III). In order to investigate the possibility that lysogeny of the recipient increased the transfer frequency, cultures of strain 1030 made lysogenic for the transducing phage from either 
strain 609 or 6936 were used as recipients in mixed-culture experiments with culture 6936 (CPNS) as the donor. Transfer of the element (CPNS) to the non-lysogenic culture $1030^{\circ}$ occurred at $4.9 \times 10^{-8}$ but the frequency of transfer to both lysogenic derivatives was $3.0 \times 10^{-5}$. The proportions of donor and recipient after incubation for $24 \mathrm{~h}$ in these cultures was not affected by the presence of prophage in strain 1030.

\section{Discussion}

Evidence has been presented here for the existence of a plasmid, in a recently isolated strain of $S$. aureus $(\mathrm{M} 7)$, determining production of penicillinase and resistance to cadmium ions, neomycin, kanamycin and streptomycin. After loss of the plasmid (CPNS), strain M7 was still resistant to kanamycin and streptomycin; this is indicative of the presence of two separate sets of genes determining resistance to streptomycin and to kanamycin in strain M7-wild. When the (CPNS) plasmid was transferred from the donor strain M7-wild, recipients harbouring fragments of the plasmid were sometimes detected. However, plasmid (CPNS) was apparently always transferred intact from donors other than from strain M7-wild. Because transfer from M7 still occurred at low frequency when the (CPNS) plasmid had been transferred back into $\mathrm{M} 7$ after passage through several other strains, it can be concluded that the low frequency of transfer and fragmentation of this element are probable functions of strain $\mathrm{M} 7$. Other antibiotic-resistance determinants in this strain may affect the stability of (CPNS) as can be demonstrated for some other plasmids that have been introduced simultaneously into a single cell (Lacey and Chopra, 1974).

That the plasmid (CPNS) can fragment suggests that it might have arisen by the reverse of such a process, e.g., recombination between a penicillinase plasmid (CPNS), i.e., resistance to cadmium ions, and sensitivity to arsenate streptomycin. Possibly relevant to this is that another staphylococcal plasmid that might have arisen by recombination, the PF plasmid (Lacey and Grinsted 1972), contains a pattern of resistances to heavy metals identical to that of plasmid (CPNS), i.e., resistance to cadmium ions, and sensitivity to arsenate and mercury. If plasmid (CPNS) is indeed a result of such an event, there is little evidence to suggest whether recombination had occurred within strain M7 or had been acquired by a transfer event, the element having arisen in another strain.

The fragments (NS), and (CP) of the element (CPNS) have different properties in strain 6936. Fragment (NS) shows features typical of chromosomal genes including inability to confer immunity to an incoming intact (CPNS) element. By contrast (CP) retains plasmid features including the ability to confer immunity to the introduction of (CPNS). The spontaneous transition of plasmid genes into the chromosome has rarely been described in S. aureus. Asheshov (1969) found, in one strain, a reduplication of chromosomal penicillinase genes on storage, one copy becoming plasmid; therefore the reverse process might occur. Novick (1967) forced integration of plasmid-mediated 
erythromycin resistance into the chromosome after prolonged ultraviolet irradiation of a transducing lysate, and Novick and Bouanchaud (1971) described similar events for penicillinase production. There is also some evidence for the integration of plasmid-mediated tetracycline resistance in methicilline-resistant staphylococci in nature (Lacey, 1975). Although these plasmid/chromosome transitions may be uncommon, their evolutionary significance may be profound because of their expected irreversibility, i.e., the genome of that cell is altered stably. We have been unable to reverse the process of chromosomal integration of (NS): transduction of genes from a strain containing both fragments $(6936(\mathrm{SN})(\mathrm{Cp}))$ always results in either the (NS) or the (CP) determinant being acquired by the recipient.

The frequency of transfer of (CPNS) and its fragments in mixed-culture corresponds well with the relative frequencies of transduction from cell-free lysates (table III). This correlation is consistent with the proposal that the transferring vehicle in mixed-culture mating is a defective phage particle (Lacey, 1971 $b$ and 1975). This study also shows how the particular donor, plasmid and recipient can alter the transfer frequency. Regions of a prophage carried by the recipient are known to be essential for transduction of methicillin resistance from cell-free lysates (Cohen, Sweeney and Basu, 1977). This phenomenon was also seen in this study in mixed-culture transfers, and is consistent with the view that that the transferring vehicles of these two processes are essentially the same.

The extremely high frequency of spontaneous transfer and of transduction of (CPNS) in certain lysates suggests that some specific association may exist between plasmid (CPNS) and the transducing phage in strain 6936. Examination of the homology of DNA between this phage and plasmid might reveal such information, and indeed might be relevant to the evolutionary relationship between staphylococcal phages and plasmids.

\section{SUMMARY}

A strain of Staphylococcus aureus (M7) contains a transmissible element determining production of penicillinase, and resistance to cadmium ions, neomycin, streptomycin and kanamycin (CPNS). This element was transferred either in toto or in fragments at low frequency from strain M7. The fragment (NS) possesses features typical of chromosomal genes and the fragment (CP), like (CPNS) itself, exhibits plasmid features. The element (CPNS) is transferred in mixed culture at high frequency, up to $10^{-3}$, between other strains of staphylococci. Lysogenisation of the recipient increases the frequency of transfer. The frequency of transduction of (CPNS), (CP) and (NS) from cell-free lysates corresponds well with the transfer frequency of each of these elements in mixed culture. A possible mechanism for the evolution of (CPNS) is discussed.

\section{REFERENCES}

AsHeshov, E. H. 1969. The genetics of penicillinase production in Staphylococcus aureus strain PS 80. J. gen. Microbiol., 59, 289. 
CoHen, S., Sweeney, H. M. AND Basu, S. K. 1977. Mutations in prophage $\phi$ II that impair the transducibility of their Staphylococcus aureus lysogens for methicillin resistance. J. Bact., 129, 237.

LACEY, R. W. $1971 a$. Transfer of tetracyline resistance between strains of Staphylococcus aureus in mixed cultures. J. gen. Microbiol. 69, 229.

LACEY, R. W. 1971b. High-frequency transfer of neomycin resistance between naturally occurring strains of Staphylococcus aureus. J. med. Microbiol., 4, 73.

LACEY, R. W. 1975. Antibiotic resistance plasmids of Staphylococcus aureus and their clinical importance. Bact. Rev., 39, 1.

LACEY, R. W. AND CHOPRA, I. 1974. Genetic studies of a multi-resistant strain of Staphylococcus aureus constructed in vitro. J. med. Microbiol., 7, 285.

LACEY, R. W. AND GRINSTED, J. 1972. Linkage of fusidic acid resistance to the penicillinase plasmid in Staphylococcus aureus. J. gen. Microbiol., 73, 501.

Novick, R. P. 1967. Penicillinase plasmids of Staphylococcus aureus. Fedn Proc. Fedn Am. Socs. exp. Biol. 29, 27.

Novick, R. P. AND BouANCHAUd D. 1971. Extrachromosomal nature of drug resistance in Staphylococcus aureus. Ann. N.Y. Acad. Sci., 182, 279. 